UFRJ-IF-FPC-006/96

\title{
Local Charged States of the Gauge Field in Three Dimensional Maxwell-Type Theories
}

\author{
E.C.MARINO \\ Instituto de Física \\ Universidade Federal do Rio de Janeiro \\ Cx.P. 68528 \\ Rio de Janeiro RJ 21945-970 \\ Brasil
}

\begin{abstract}
Gauge invariant local creation operators of charged states are introduced and studied in pure gauge theories of the Maxwell type in $2+1 \mathrm{D}$. These states are usually unphysical because of the subsidiary condition imposed on the physical subspace by Gauss' law. A dual Maxwell theory which possesses a topological electric charge is introduced. Pure Electrodynamics lies in the sector where the topological charge identically vanishes. Charge bearing operators fully expressed in terms of the gauge field, however, can create physical states in the nontrivial topological sectors which thereby generalize QED. An order disorder structure exists relating the charged operators and the magnetic flux creating (vortex) operators, both through commutation rules and correlation functions. The relevance of this structure for bosonization in $2+1 \mathrm{D}$ is discussed.
\end{abstract}

* Work supported in part by CNPq-Brazilian National Research Council. E-Mail address: marino@if.ufrj.br 


\section{1) Introduction}

A rather interesting problem in Quantum Field Theory is the obtainement of the operators creating the quantum states carrying the topological charge of a certain system. In three dimensional space, the topological charge is the magnetic flux along the spatial plane and this problem has been solved years ago [1, 2, 3, 4, 5]. The underlying framework for this is an abelian gauge theory in $2+1 \mathrm{D}$. Given these topological charge creating operators (vortex operators), there is usually an order-disorder duality structure relating them to dual operators carrying another conserved charge which in $2+1$ dimensions is the electric charge. The construction of this second type of operators is also an appealing problem. Moreover, it is known that this type of order-disorder structure is responsible for bosonization in 1+1D [6, 7] and also in higher dimensions [8, 9].

A charge bearing operator dual to the vortex operator was introduced recently in Maxwell-type theories [10]. That construction, however, besides being rather cumbersome, has the disadvantage of being non gauge invariant. In addition the states created by that operators did not satisfy the subsidiary condition imposed by Gauss' law on the physical states. More recently charge bearing local gauge invariant operators were obtained in Chern-Simons theory, both pure and coupled to fermions [11. In the present work we introduce local gauge invariant charge bearing operators in Maxwell-type theories in $2+1 \mathrm{D}$ and make a detailed study of their properties and correlation functions. The present construction is much simpler and transparent than that of [10]. We also introduce a theory which is dual to Maxwell QED, in which the electric charge appears as the topological charge. Pure Electrodynamics corresponds to the sector were the toplogical charge is identically zero. The nontrivial sectors consist in a generalization of QED. An analogous system was introduced recently in $3+1 \mathrm{D}$ [12]. In this theory, the charge carrying operators dual to the vortex field do belong to the physical subspace.

In Section 2, we introduce the charge carrying $\sigma$-operators in Maxwell theory and evaluate its commutators with the charge and with the magnetic flux carrying vortex 
$\mu$-operator. The locality of its correlation functions is also demonstrated in general. In Section 3, we evaluate the correlation functions of $\sigma$ as well as the mixed $\sigma-\mu$ correlation functions both in Maxwell theory and in a nonlocal version of it which appeared in the bosonization of a free massless fermion field in $2+1 \mathrm{D}$ [8]. This theory, given by (3.17), was also shown to describe the real electromagnetic interaction of four-dimensional charged particles constrained to move on a plane [13. In Section 4, we introduce the dual Maxwell theory and the generalization of it containing nontrivial topological sectors. The charge bearing operators are introduced and their correlation functions, explicitly evaluated. Two Appendixes are also included.

\section{2) The $\sigma$ Operator}

\section{1) Correlation Functions}

Let us start with Maxwell theory and introduce the operator $\sigma$ which will create the charged states of the gauge field and whose correlation functions are going to be local. For these, inspired in the construction of similar operators in Chern-Simons theory 11], we write

$$
<\sigma(x) \sigma^{\dagger}(y)>=Z^{-1} \int D A_{\mu} \exp \left\{-\int d^{3} z\left[\frac{1}{4} F_{\mu \nu} F^{\mu \nu}+F_{\mu \nu} \tilde{C}^{\mu \nu}+\frac{1}{2} \tilde{C}_{\mu \nu} \tilde{C}^{\mu \nu}\right]\right\}
$$

where $\tilde{C}_{\mu \nu}=\tilde{C}_{\mu \nu}(z ; x)-\tilde{C}_{\mu \nu}(z ; y)$ with $\tilde{C}_{\mu \nu}(z, x)$ given by

$$
\tilde{C}_{\mu \nu}(z ; x)=\partial_{\nu} \tilde{C}_{\mu}(z ; x)
$$

with

$$
\tilde{C}_{\mu}(z ; x)=i a \int_{x, L}^{\infty} d \xi_{\mu}\left[\frac{1}{-\square}\right](z-\xi)
$$

Here $\left[\frac{1}{-\square}\right]=\frac{1}{4 \pi|x|}$ is the Fourier transform of $1 / k^{2}$. As in previous cases [11, [3] the second term in (2.1) corresponds to the operator itself and the last term is a renormalization factor which removes unphysical parts and makes (2.1) independent of the line $L$ appearing in the definition of the "external field" $\tilde{C}_{\mu \nu}$ in (2.2). This will be shown explicitly below. 


\section{2) Commutation Rules}

From (2.1) we can extract the form of $\sigma$, namely

$$
\begin{gathered}
\sigma(x)=\exp \left\{-i \int d^{3} z \tilde{C}_{\mu \nu} F^{\mu \nu}\right\} \\
\sigma(x)=\exp \left\{i a \int_{x, L}^{\infty} d \xi_{\beta} \int d^{3} z\left[\frac{1}{-\square}\right](\xi-z) \partial_{\alpha} F^{\alpha \beta}(z)\right\} \\
\sigma(x)=\exp \left\{i a \int_{x, L}^{\infty} d \xi^{i} A_{i}(\xi)+\int d^{3} z\left[\frac{1}{-\square}\right](x-z) \partial_{\alpha} A^{\alpha}(z)\right\}
\end{gathered}
$$

We see that $\sigma$ is gauge invariant and the first part of it is the Wilson line.

Let us consider now the commutator of $\sigma$ with the charge density operator $j^{0}=$ $\partial_{i} E^{i}$. We can always choose the gauge in such a way that the second part commutes with $j^{0}$. The first part immediately gives

$$
\left[j^{0}(x), \sigma(y)\right]=a \sigma \delta^{2}(x-y)
$$

showing that $\sigma$ carries $a$ units of charge. The states created by $\sigma$, however, are not physical because by virtue of (2.4) they do not satisfy the subsidiary condition imposed by Gauss' law on the physical states, namely $Q \mid$ phys $>=0$. We will introduce in Section 3 a theory that generalizes Maxwell and in which the $\sigma$ states become physical.

It will be interesting to verify the commutation rules of $\sigma$ with the vortex creation operator introduced in [1, 3, 10], which is given by

$$
\mu(x) \exp \left\{-i b \int_{T_{L(x)}} d^{2} \xi F^{i 0}\left(\xi, x^{0}\right) \partial_{i} \arg (\xi-x)\right\}
$$

In this expression, $T_{L(x)}$ is the surface in Fig. 1, which consists of a plane of which a wedge containing the line $L(x)$, going from $x$ to infinity has been taken out. This line corresponds to the cut of the arg function appearing in (2.5). At the end we must take the cutoff $\delta$

Again, the second part of (2.3) commutes with $\mu$ and we immediately obtain

$$
\mu(x) \sigma(y)=\sigma(y) \mu(x) \exp \left\{-i a b \int_{y, L(y)}^{\infty} d \eta^{i} \int_{T_{L(x)}} d^{2} \xi \partial_{i}^{(\eta)} \arg (\eta-x) \delta^{2}(\xi-\eta)\right\}
$$




$$
\mu(x) \sigma(y)=\sigma(y) \mu(x) \exp \{i a b \arg (\vec{y}-\vec{x})\}
$$

provided we choose the line $L(y)$ to belong to $T_{L(x)}$. This is precisely the correct dual algebra satisfied by the operator $\mu$ with charged operators [2, 33, 10].

\section{3) Locality}

Let us demonstrate now on general grounds, the line independence (locality) of (2.1). For this, let us perform the following change of functional integration variable in (2.1)

$$
A_{\mu} \rightarrow A_{\mu}+\Omega_{\mu}
$$

with

$$
\Omega_{\mu}=a \oint_{L^{\prime}-L} d \xi_{\mu}\left[\frac{1}{-\square}\right](z-\xi)
$$

where $L^{\prime}$ is an arbitrary line going from $x$ to infinity. Under this transformation we have

$$
F_{\mu \nu} \rightarrow F_{\mu \nu}+\tilde{C}_{\mu \nu}\left(L^{\prime}-L\right)-\tilde{C}_{\nu \mu}\left(L^{\prime}-L\right)
$$

In Appendix A we show that under (2.7) and (2.8) the exponent in (2.1), namely

$$
\mathcal{L}\left[F_{\mu \nu}, \tilde{C}_{\mu \nu}(L)\right]=\frac{1}{4} F_{\mu \nu} F^{\mu \nu}+F_{\mu \nu} \tilde{C}^{\mu \nu}+\frac{1}{2} \tilde{C}_{\mu \nu} \tilde{C}^{\mu \nu}
$$

gets transformed as

$$
\mathcal{L}\left[F_{\mu \nu}, \tilde{C}_{\mu \nu}(L)\right] \rightarrow \mathcal{L}\left[F_{\mu \nu}, \tilde{C}_{\mu \nu}\left(L^{\prime}\right)\right]
$$

This shows that the correlation function $<\sigma(x) \sigma^{\dagger}(y)>$ is independent of the curve $L$ appearing in the definition of the operator $\sigma$, being therefore local.

\section{3) Correlation Functions}

\section{1) Maxwell Theory}

Let us explicitly evaluate in this Section the euclidean correlation functions involving the operator $\sigma$ in the case of pure Maxwell theory. From (2.1) we obtain, after performing the quadractic functional integral,

$$
<\sigma \sigma^{\dagger}>=\exp \left\{\frac{1}{2} \int d^{3} z d^{3} z^{\prime} \tilde{C}_{\mu \nu}(z) \tilde{C}_{\alpha \beta}\left(z^{\prime}\right) F_{\sigma}^{\mu \nu}(z) F_{\lambda}^{\alpha \beta}\left(z^{\prime}\right) D^{\sigma \lambda}\left(z-z^{\prime}\right)-S_{L}\right\}
$$


where

$$
F_{\sigma}^{\mu \nu}(z) \equiv \partial^{\mu} \delta_{\sigma}^{\nu}-\partial^{\nu} \delta_{\sigma}^{\mu}
$$

and $D^{\sigma \lambda}$ is the euclidean propagator of the Maxwell field, given by

$$
D^{\sigma \lambda}=\left(-\square \delta^{\sigma \lambda}+\left(1-\frac{1}{\xi}\right) \partial^{\sigma} \partial^{\lambda}\right)\left[\frac{1}{(-\square)^{2}}\right]
$$

where $\xi$ is the gauge fixing parameter. $S_{L}$ in (3.1) is the $\tilde{C}_{\mu \nu}$ quadractic renormalization factor appearing in the last term of (2.1). Inserting (3.2) and (3.3) in (3.1), we see that only the gauge independent first term of (3.3) contributes to it, thus confirming the gauge invariance of the $\sigma$ correlation function. Inserting $\tilde{C}_{\mu \nu}$ in (3.1) we get

$$
\begin{gathered}
<\sigma \sigma^{\dagger}>=\exp \left\{\frac{a^{2}}{2} \sum_{i, j=1}^{2} \lambda_{i} \lambda_{j} \int_{x_{i}, L}^{\infty} d \xi^{i} \int_{x_{j}, L}^{\infty} d \eta^{j} \times\right. \\
\left.\frac{\partial_{(\xi)}^{\nu}}{-\square} \frac{\partial_{(\eta)}^{\beta}}{-\square} F_{\sigma}^{i \nu}(\xi) F_{\sigma}^{j \beta}(\eta)[-\square]\left[\frac{1}{(-\square)^{2}}\right](\xi-\eta)-S_{L}\right\}
\end{gathered}
$$

where $x_{1} \equiv x, x_{2} \equiv y, \lambda_{1} \equiv+1, \lambda_{2} \equiv-1$. Using the identity

$$
F_{\sigma}^{i \nu}(\xi) F_{\sigma}^{j \beta}(\eta) \equiv \epsilon^{i \nu \sigma} \epsilon^{j \beta \lambda}\left[\partial_{(\xi)}^{\rho} \partial_{(\eta)}^{\rho} \delta^{\sigma \lambda}-\partial_{(\xi)}^{\sigma} \partial_{(\eta)}^{\lambda}\right]
$$

in (3.4) we see that the second term vanishes because of the $\epsilon$ 's and the contribution form the first term cancels the two $\frac{1}{\square}$ pieces. Employing now the identity $\epsilon^{i \nu \sigma} \epsilon^{j \beta \sigma} \equiv \delta^{i j} \delta^{\nu \beta}-\delta^{i \beta} \delta^{\nu j}$, we see that the first term which is line dependent is identically canceled by the renormalization factor $S_{L}$. The second term is easily seen to be line independent, upon integration over $\xi$ and $\eta$. The result is

$$
<\sigma \sigma^{\dagger}>=\exp \left\{-\frac{a^{2}}{2} \sum_{i, j=1}^{2} \lambda_{i} \lambda_{j} F\left(x_{i}-x_{j}\right)\right\}
$$

where

$$
F(x)=\frac{1}{(-\square)^{2}} \equiv \mathcal{F}^{-1}\left(\frac{1}{k^{4}}\right)=\lim _{m \rightarrow \infty}\left[\frac{1}{m}-\frac{|x|}{8 \pi}\right]
$$

Here $m$ is an infrared regulator used to define the inverse Fourier transform of $\frac{1}{k^{4}}$. Introducing (3.7) in (3.6) we see that the $m$-dependent part cancels and all infrared singularities disappear. The remaining term gives

$$
<\sigma(x) \sigma^{\dagger}(y)>=\exp \left\{-\frac{a^{2}}{8 \pi}|x-y|\right\}
$$


This is the final result for the $\sigma$ correlation function. The exponential decay would indicate a massive $\mid \sigma>$ state but as we saw this is unphysical in pure Maxwell theory. The same expression as (3.8) has been obtained in 10 for the gauge invariant part of the analogous operator. The present formulation of charged operators, however, presents the advantage of being gauge invariant and much simpler.

The correlation function of the vortex operator has been evaluated in [3], giving the result

$$
<\mu \mu^{\dagger}>=\exp \left\{\frac{\pi b^{2}}{|x-y|}\right\}
$$

The large distance behavior of this indicates that $\mu$ does not create genuine vortex states in this theory.

It will be instructive now to evaluate the mixed $\sigma-\mu$ correlation function

$$
\begin{aligned}
<\sigma\left(x_{1}\right) \sigma^{\dagger}\left(x_{2}\right) \mu\left(y_{1}\right) \mu^{\dagger}\left(y_{2}\right)> & =Z^{-1} \int D A_{\mu} \exp \left\{-\int d^{3} z\left[\frac{1}{4}\left(F_{\mu \nu}+\tilde{B}_{\mu \nu}\right)\left(F^{\mu \nu}+\tilde{B}^{\mu \nu}\right)\right.\right. \\
& \left.\left.+\left(F_{\mu \nu}+\tilde{B}_{\mu \nu}\right) \tilde{C}^{\mu \nu}+\frac{1}{2} \tilde{C}_{\mu \nu} \tilde{C}^{\mu \nu}\right]\right\}
\end{aligned}
$$

The part corresponding to $\mu$ is associated with the $\tilde{B}^{\mu \nu}$ external field, which is given by [3]

$$
\tilde{B}^{\mu \nu}(z ; x)=b \int_{T_{L(x)}} d^{2} \xi^{[\mu} \partial^{\nu]} \arg (\xi-x) \delta^{3}(z-\xi)
$$

where the surface $T_{L(x)}$ was defined above. Notice that a crossed $\tilde{B}-\tilde{C}$ renormalization term appeared in order ensure locality (path independence). The above functional integral can be computed as before. The $\tilde{C}^{\mu \nu}$ - part was just evaluated above. The $\tilde{B}^{\mu \nu}$-part was calculated in [3]. The result is

$$
<\sigma\left(x_{1}\right) \sigma^{\dagger}\left(x_{2}\right) \mu\left(y_{1}\right) \mu^{\dagger}\left(y_{2}\right)>=\exp \left\{-\frac{a^{2}}{8 \pi}|x-y|+\frac{\pi b^{2}}{|x-y|}+C T\right\}
$$

where the crossed term is given by

$$
\begin{gathered}
C T=i a b \sum_{i, j=1}^{2} \lambda_{i} \lambda_{j} \int_{x_{i}, L}^{\infty} d \xi^{i} \int_{T_{L}} d^{2} \eta_{\mu} \partial_{k} \arg \left(\xi-y_{j}\right) \frac{\partial_{(\xi)}^{\rho}}{-\square} F_{\sigma}^{i \rho}(\xi) F_{\lambda}^{\mu k}(\eta) D^{\sigma \lambda}(\xi-\eta) \\
-\int d^{3} z \tilde{C}_{\mu \nu} \tilde{B}^{\mu \nu}
\end{gathered}
$$


where the last piece is the $\tilde{B}-\tilde{C}$ term appearing in (3.10). Again only the gauge invariant first term of (3.3) contributes to (3.13). Using the identity (3.5), also here we find that the second term of (3.5) vanishes. The first term of (3.5) contains two parts: one vanishes because $d \xi^{i} \perp d^{2} \eta^{j}$, the other one gives

$$
C T=-i a b \sum_{i, j=1}^{2} \lambda_{i} \lambda_{j} \int_{T_{L}} d^{2} \eta^{\mu} \arg \left(\eta-y_{j}\right) \partial_{\mu}\left[\frac{1}{-\square}\right]\left(\eta-x_{i}\right)-\int d^{3} z \tilde{C}_{\mu \nu} \tilde{B}^{\mu \nu}
$$

In Appendix B we show that

$$
C T=A\left(x_{i}-y_{j}\right) \equiv n \arg \left(x_{i}-y_{j}\right)
$$

where $n=0, \pm 1, \ldots$. We see that $C T$ is zero modulo a function $\arg \left(x_{i}-y_{j}\right)$. We have therefore

$$
\begin{aligned}
<\sigma\left(x_{1}\right) \sigma^{\dagger}\left(x_{2}\right) \mu\left(y_{1}\right) \mu^{\dagger}\left(y_{2}\right)> & =\exp \left\{-\frac{a^{2}}{8 \pi}\left|x_{1}-x_{2}\right|+\frac{\pi b^{2}}{\left|y_{1}-y_{2}\right|}+A\left(x_{1}-y_{1}\right)+A\left(x_{1}-y_{2}\right)\right. \\
& \left.+A\left(x_{2}-y_{1}\right)+A\left(x_{2}-y_{2}\right)\right\}
\end{aligned}
$$

The ambiguities contained in the $A$-functions are nothing but a reflex of the nontrivial commutation relations between $\sigma$ 's and $\mu$ 's on the left hand side of (3.22) [6, 14]. The various possible orderings of the operators correspond to the different possibilities of arg-functions in the $A$ 's. This is a beautiful example, actually the first one in $2+1 \mathrm{D}$, of how the functional integral can correctly describe the nontrivial algebra of operators, even though the integration is performed over bosonic fields only.

\section{2) Nonlocal Maxwell Theory}

One can easily generalize the method for the evaluation of correlation functions of $\sigma$ and $\mu$ operators for the nonlocal generalization of the Maxwell theory given by

$$
\mathcal{L}^{\prime}=-\frac{1}{4} F^{\mu \nu}\left[\frac{1}{(-\square)^{1 / 2}}\right] F_{\mu \nu}
$$

This theory was shown to describe the true electromagnetic interaction of threedimensional particles constrained to move on a plane [13]. It also appeared in the bosonization of the free massless fermion in $2+1 \mathrm{D}[\mathrm{B}]$. 
In this case, the $\sigma$ and $\mu$ operators would be described by external fields analog to (2.2) and (3.19), given respectively by

$$
\tilde{C}_{\mu}(z ; x)=i a \int_{x, L}^{\infty} d \xi_{\mu}\left[\frac{1}{(-\square)^{1 / 2}}\right](z-\xi)
$$

and

$$
\tilde{B}^{\mu \nu}(z ; x)=b \int_{T(L)} d^{2} \xi^{[\mu} \partial^{\nu]} \arg (\xi-x)\left[\frac{1}{(-\square)^{1 / 2}}\right](z-\xi)
$$

with the obvious modification of the renormalization factors.

The quantization of theories described by lagrangians of the type of (3.17) has been performed in full detail in [15. One can show that $\sigma$ creates states carrying the charge $j^{0}=\partial_{i} \Pi^{i}$. Again, however, the $0^{t h}$ component of the field equation corresponding to (3.17), namely $\partial_{i} \Pi^{i}=0$ must be imposed as a constraint over the physical states, implying that the $\mid \sigma>$ states will also be unphysical here.

The evaluation of the correlation functions is entirely analogous to the case of pure Maxwell theory and we just show the results

$$
\begin{aligned}
& <\sigma(x) \sigma^{\dagger}(y)>=\frac{1}{|x-y|^{a^{2}}} \\
& <\mu(x) \mu^{\dagger}(y)>=\frac{1}{|x-y|^{b^{2}}}
\end{aligned}
$$

and

$$
\begin{aligned}
<\sigma\left(x_{1}\right) \sigma^{\dagger}\left(x_{2}\right) \mu\left(y_{1}\right) \mu^{\dagger}\left(y_{2}\right)>= & \frac{1}{\left|x_{1}-x_{2}\right|^{2}} \frac{1}{\left|y_{1}-y_{2}\right|^{b^{2}}} \exp \left\{A\left(x_{1}-y_{1}\right)+A\left(x_{1}-y_{2}\right)+\right. \\
& \left.A\left(x_{2}-y_{1}\right)+A\left(x_{2}-y_{2}\right)\right\}
\end{aligned}
$$

Notice that $a$ and $b$ are dimensionless now. Again we have the $A(x-y)$ factors in the mixed function, indicating that the $\sigma$ and $\mu$ operators will have the same commutation relations as in the Maxwell theory. This confirms the result of the quantization method developed in 15. 


\section{4) The Dual Theory}

We saw in Section 2 that the charged states created by $\sigma$ were unphysical in pure Maxwell theory. We will introduce now a generalization of this theory in which these charged $\sigma$-states do exist in the physical space.

First of all, let us introduce a vector gauge field $W_{\mu}$, in terms of which we can express the vacuum functional of Maxwell theory as the following euclidean functional integral

$$
\begin{gathered}
Z_{A}=\int D A_{\mu} \exp \left\{-\int d^{3} z \frac{1}{4} F^{\mu \nu} F_{\mu \nu}\right\}= \\
=Z_{0}^{-1} \int D W_{\mu} \exp \left\{-\int d^{3} z\left[\frac{1}{4} W^{\mu \nu}\left[\frac{1}{-\square}\right] W_{\mu \nu}+i \epsilon^{\mu \alpha \beta} W_{\mu} \partial_{\alpha} A_{\beta}\right]+\mathcal{L}_{G F}\right\}
\end{gathered}
$$

The field equation associated to the lagrangian of the $W_{\mu}-A_{\mu}$ theory

$$
\mathcal{L}_{W A}=\frac{1}{4} W^{\mu \nu}\left[\frac{1}{-\square}\right] W_{\mu \nu}-\epsilon^{\mu \alpha \beta} W_{\mu} \partial_{\alpha} A_{\beta}
$$

can be written as

$$
\partial_{\nu} F^{\mu \nu}=\epsilon^{\mu \alpha \beta} \partial_{\alpha} W_{\beta}
$$

We see that the topological current of the $W_{\mu}$-field, namely $J^{\mu}=\epsilon^{\mu \alpha \beta} \partial_{\alpha} W_{\beta}$ becames the source of the electromagnetic field, that is, an electric current. If we integrate over $A_{\mu}$ in (4.1), however, we get

$$
Z_{A}=Z_{0}^{-1} \int D W_{\mu} \exp \left\{-\int d^{3} z\left[\frac{1}{4} W^{\mu \nu}\left[\frac{1}{-\square}\right] W_{\mu \nu}+\mathcal{L}_{G F}\right]\right\} \delta\left[\epsilon^{\mu \alpha \beta} \partial_{\alpha} W_{\beta}\right]
$$

We conclude that pure Maxwell theory can be described by the pure $W_{\mu}$ theory with the constraint that we should be in the sector where the topological charge (electric charge) is identically equal to zero.

Let us consider now the theory given by

$$
\mathcal{L}_{W}=-\frac{1}{4} W^{\mu \nu}\left[\frac{1}{-\square}\right] W_{\mu \nu}=\frac{1}{2} J^{\mu}\left[\frac{1}{-\square}\right] J_{\mu}
$$

where the constraint $J^{\mu} \equiv 0$ has been relaxed. Of course this is no longer Maxwell theory, but a generalization of it where we can have physical states with a nonzero topological charge. As we can infer from the second part of (4.5) the interaction 
of these charges will be identical to that of usual charges interacting through the electromagnetic field.

Let us introduce now the operator $\tilde{\sigma}$ which is going to create the states carrying topological charge (electric charge) in the theory described by $\mathcal{L}_{W}$. Consider

$$
\begin{gathered}
\tilde{\sigma}(x)=\exp \left\{i \frac{a}{2} \int_{x, L}^{\infty} d \xi_{\lambda} \epsilon^{\lambda \mu \nu}\left[\frac{W^{\mu \nu}}{-\square}\right]\right\} \\
\tilde{\sigma}(x)=\exp \left\{i a \int_{x, L}^{\infty} d \xi^{i} \epsilon^{i j} \Pi^{j}\right\}
\end{gathered}
$$

where $\Pi^{i}=\frac{W^{0 i}}{-\square}$. Observe that if we use the field equation (4.3) in (4.6) we can see that $\tilde{\sigma}=\sigma$, as given by $(2.3)$.

The quantization of theories of the type described by (4.5) has been carefully studied in [15]. One can show that

$$
\left[J^{0}(x), \tilde{\sigma}(y)\right]=a \tilde{\sigma}(y) \delta^{2}(x-y)
$$

indicating that indeed $\tilde{\sigma}$ does create states with $a$ units of charge.

Let us consider now the correlation function of $\tilde{\sigma}$. This can be written as

$$
<\tilde{\sigma}(x) \tilde{\sigma}^{\dagger}(y)>=Z_{0}^{-1} \int D W_{\mu} \exp \left\{-\int d^{3} z\left[\frac{1}{4}\left(W^{\mu \nu}+\bar{B}^{\mu \nu}\right)\left[\frac{1}{-\square}\right]\left(W_{\mu \nu}+\bar{B}_{\mu \nu}\right)\right]\right\}
$$

where $\bar{B}^{\mu \nu}=\bar{B}^{\mu \nu}(z ; x)-\bar{B}^{\mu \nu}(z ; y)$ with

$$
\bar{B}^{\mu \nu}(z ; x)=a \int_{x, L}^{\infty} d \xi_{\lambda} \epsilon^{\lambda \mu \nu} \delta^{3}(z-\xi)
$$

The crossed $W-\bar{B}$ term is easily identified with the $\tilde{\sigma}$ operators (4.6). The $\bar{B}-\bar{B}$ term, as before is a renormalization factor which renders (4.8) path independent. The locality of (4.8) can be demonstrated in general by making a transformation similar to (2.7) but with $\Omega_{\mu}$ replaced by [3]

$$
\tilde{\Omega}_{\mu}=a \int_{S\left(L^{\prime}-L\right)} d \xi_{\mu} \delta^{3}(z-\xi)
$$

where $S\left(L^{\prime}-L\right)$ is an arbitrary surface bounded by $L$ and a general curve $L^{\prime}$. 
In order to evaluate $<\tilde{\sigma} \tilde{\sigma}^{\dagger}>$ we insert (B.1) in (4.8) and integrate over $W_{\mu}$, obtaining

$$
\begin{gathered}
<\tilde{\sigma}(x) \tilde{\sigma}^{\dagger}(y)>=\exp \left\{\frac{a^{2}}{2} \sum_{i, j=1}^{2} \lambda_{i} \lambda_{j} \int_{x_{i}, L} d \xi^{i} \int_{x_{j}, L} d \eta^{j} \times\right. \\
\left.\epsilon^{i \mu \alpha} \frac{\partial_{\mu}^{(\xi)}}{-\square} \epsilon^{j \nu \beta} \frac{\partial_{\nu}^{(\eta)}}{-\square}\left[-\square \delta^{\alpha \beta}+\left(1-\frac{1}{\xi}\right) \partial^{\alpha} \partial^{\beta}\right]\left[\frac{1}{-\square}\right]-S_{L}\right\}
\end{gathered}
$$

In this expression, $S_{L}$ is the $\bar{B}-\bar{B}$ term of (4.8) and the expression between brackets is the euclidean propagator of the $W_{\mu}$ field, corresponding to (4.5). We use the same convention for the $\lambda$ 's as before. Only the gauge invariant first part of the propagator contributes in (4.11). The rest identically vanishes. Using the identity for the $\epsilon$ 's appearing after (3.5) we get two terms. One of them is path dependent and is identically canceled by $S_{L}$. The remaining term is path independent and gives an expression identical to (3.6). We therefore obtain

$$
<\tilde{\sigma}(x) \tilde{\sigma}^{\dagger}(y)>=\exp \left\{-\frac{a^{2}}{8 \pi}|x-y|\right\}
$$

The massive $\mid \sigma>$ states are no longer forbidden by the condition to be imposed on the physical subspace. We should remark, however that they will not be asymptotic, because of the short distance behavior of the correlation function (4.12).

It is interesting to observe that also in $3+1 \mathrm{D}$ a theory dual to electrodynamics was found [12] in complete analogy to what we did here. This theory is formulated in terms of an antisymmetric tensor gauge field. In the generalization where the zero topological charge constraint is relaxed we find massless charged states which can be asymptotic for a certain value of the charge [12].

\section{5) Final Comments}

We have seen how to construct creation operators of charged states, which are expressed only in terms of the gauge field, in three dimensional theories whose lagrangian is quadractic in the field intensity tensor. This charge bearing gauge invariant operators are always dual to the magnetic flux carrying operator (vortex operator) in each of the theories considered. Only in the theory introduced in Section 3, however, 
which generalizes the simple Maxwell case, by the introduction of nontrivial topological sectors, these charged states are physical. This theory is completely analogous to the one investigated in [12] in connection to four-dimensional QED. Even in the cases where the states are unphysical, however, they are interesting and worth to be investigated, for the following reason. Putting together the charged operators introduced here and the magnetic flux operators introduced in [1, 2, 3] we have a complete picture of duality in $2+1 \mathrm{D}$. This has the same structure as the one found in $1+1 \mathrm{D}$, involving scalar fields [7, 6]. The novelty here is that since we have gauge theories underlying the construction, the states created by the corresponding operators are not always physical. It is known [6] that in $1+1 \mathrm{D}$, this structure is responsible for the bosonization of fermions hence it is quite natural to expect that this is also true in higher dimensions. The case of a free massless fermion field in $2+1 \mathrm{D}$ has been considered in [8]. Some general results have been obtained more recently in [9]. We are presently investigating the application of the dual operators we found in $2+1 \mathrm{D}$ in connection with the method of bosonization.

\section{A) Appendix A}

Let us demonstrate here Eq.(2.10) and thereby establish the path independence of (2.1). Inserting (2.8) in (2.9) we get

$$
\mathcal{L}\left[F_{\mu \nu}, \tilde{C}_{\mu \nu}(L)\right] \rightarrow \mathcal{L}\left[F_{\mu \nu}, \tilde{C}_{\mu \nu}(L)\right]+\Delta \mathcal{L}\left[F_{\mu \nu}, \tilde{C}_{\mu \nu}(L)\right]
$$

where

$$
\begin{gathered}
\Delta \mathcal{L}\left[F_{\mu \nu}, \tilde{C}_{\mu \nu}(L)\right]=\frac{1}{2} \tilde{C}_{\mu \nu} \tilde{C}_{\mu \nu}\left(L L+L^{\prime} L^{\prime}-2 L L^{\prime}\right)-\frac{1}{2} \tilde{C}_{\mu \nu} \tilde{C}_{\nu \mu}\left(L L+L^{\prime} L^{\prime}-2 L L^{\prime}\right) \\
+\tilde{C}_{\mu \nu}(L) \tilde{C}_{\mu \nu}\left(L^{\prime}-L\right)-\tilde{C}_{\mu \nu}(L) \tilde{C}_{\nu \mu}\left(L^{\prime}-L\right)++\tilde{C}_{\mu \nu}\left(L^{\prime}-L\right) F^{\mu \nu}
\end{gathered}
$$

In the above expression we have schematically represented the $L$ and $L^{\prime}$ dependence in an obvious way. The crossed $L L^{\prime}$ terms cancel. The $L L$ and $L^{\prime} L^{\prime} \mu \nu-\nu \mu$ terms are of the form

$$
\frac{1}{2} \tilde{C}_{\mu \nu}(L) \tilde{C}_{\nu \mu}(L)-\left(L \leftrightarrow L^{\prime}\right)=
$$




$$
=\int_{z, L}^{\infty} d \xi_{\mu} \int_{z^{\prime}, L}^{\infty} d \eta_{\nu} \partial_{(\xi)}^{\mu} \partial_{(\eta)}^{\nu} F(\xi-\eta)-\left(L \leftrightarrow L^{\prime}\right)=0
$$

This is zero because the above integral is clearly independent of the curve $L$. Now, inserting the remaining terms of (A.2) in (A.1) we see that the $\tilde{C}_{\mu \nu} F^{\mu \nu}$ and $\tilde{C}_{\mu \nu} \tilde{C}^{\mu \nu}$ terms in (A.2) will combine with the corresponding ones in 2.9) thereby exchanging $L$ for $L^{\prime}$ in $\mathcal{L}\left[F_{\mu \nu}, \tilde{C}_{\mu \nu}(L)\right]$. This establishes Eq.(2.10).

\section{B) Appendix B}

Let us demonstrate here Eq.(3.15). According to Fig. 2, the first part of (3.15) is given by

$$
\begin{gathered}
I=-i a b \int_{T_{L\left(y_{j}\right)}} d^{2} \xi^{\mu} \arg \left(\xi-y_{j}\right) \partial_{\mu}\left[\frac{1}{4 \pi\left|\xi-x_{i}\right|}\right]= \\
=-i a b \frac{H}{4 \pi} \int_{0}^{\infty} d r r \int_{0}^{2 \pi} d \theta \frac{\theta}{\left[D^{2}+r^{2}-2 L r \cos (\theta-\alpha)\right]^{3 / 2}}= \\
=-i a b \frac{H}{4 \pi} \int_{0}^{\infty} d r r \int_{-\alpha}^{2 \pi-\alpha} d \omega \frac{\omega+\alpha}{\left[D^{2}+r^{2}-2 L r \cos \omega\right]^{3 / 2}}
\end{gathered}
$$

Now,

$$
\begin{gathered}
I^{\prime}=\int_{T_{L\left(y_{j}\right)}} d^{2} \xi^{\mu} \partial_{\mu}\left[\frac{1}{4 \pi\left|\xi-x_{i}\right|}\right]=\int_{T_{L\left(y_{j}\right)}} \frac{\overrightarrow{d S} \cdot \hat{R}}{4 \pi R^{2}}=\frac{H}{4 \pi} \int_{0}^{\infty} d r r \int_{-\alpha}^{2 \pi-\alpha} d \omega \frac{1}{\left[D^{2}+r^{2}-2 L r \cos \omega\right]^{3 / 2}} \\
=\frac{\Omega\left(x_{i} ; T_{L\left(y_{j}\right)}\right)}{4 \pi}
\end{gathered}
$$

where $\Omega\left(x_{i} ; T_{L\left(y_{j}\right)}\right)$ is the solid angle formed by $x_{i}$ and the surface $T_{L\left(y_{j}\right)}$. This is defined up to $4 \pi$ factors. Actually, in the limit when we take out the cutoff from $T_{L\left(y_{j}\right)}$ we have $\Omega\left(x_{i} ; T_{L\left(y_{j}\right)}\right)=2 \pi+n 4 \pi$ where $n$ is an arbitrary integer.

Comparing $(\overline{B .2})$ and $(\underline{B} .1)$ we see that

$I=-i a b \arg \left(x_{i}-y_{j}\right) \frac{\Omega\left(x_{i} ; T_{L\left(y_{j}\right)}\right)}{4 \pi}-i a b \frac{H}{4 \pi} \int_{0}^{\infty} d r r \int_{-\alpha}^{2 \pi-\alpha} d \omega \frac{\omega}{\left[D^{2}+r^{2}-2 L r \cos \omega\right]^{3 / 2}}$

Using now (3.19) and (2.2), we see that the second part of (3.15) is precisely $-I$. Hence $(\mathbb{B} .3)$ is canceled and in particular the line(cut)-dependent second piece. Since the first term of $(\mathbb{B} .3)$, however, is ambiguously defined we can always choose 
the $n$-integers in the $\Omega\left(x_{i} ; T_{L\left(y_{j}\right)}\right)$ 's in such a way that there always remains a factor $A\left(x_{i}-y_{j}\right) \equiv n \arg \left(x_{i}-y_{j}\right)$ for arbitrary $n$. This proves (3.15). Each prescription for $n$ corresponds to a particular ordering of operators on the left hand side of (3.22).

\section{References}

[1] J.Polchinski, Nucl. Phys. B179 (1981) 509

[2] E.C.Marino, Phys. Rev. D38 (1988) 3194

[3] E.C.Marino, Int. J. Mod. Phys. A10 (1995) 4311

[4] J.Fröhlich and P.A.Marchetti, Lett. Math. Phys. 16 (1988) 347; Commun. Math. Phys. 121 (1989) 177; J.Fröhlich, F.Gabiani and P.A.Marchetti, in "Proceedings, Banff Summer School in Theoretical Physics" (H.C.Lee, Ed.)

[5] G.W.Semenoff and P.Sodano, Nucl. Phys. B328 (1989) 753; M.Lüscher, Nucl. Phys. B326 (1989) 557; R.Jackiw and S.Y.Pi, Phys. Rev. D42 (1990) 3500; A.Kovner, B.Rosenstein and D.Eliezer, Nucl. Phys. B350 (1991) 325; Mod. Phys. Lett. A5 (1990) 2733; V.F.Müller, Z.Phys. C51 (1991) 665

[6] E.C.Marino and J.A.Swieca, Nucl. Phys. B170 [FS1] (1980) 175

[7] S.Coleman Phys. Rev. D11 (1975) 2088; S.Mandelstam, Phys. Rev. D11 (1975) 3026

[8] E.C.Marino, Phys. Lett. B263 (1991) 63

[9] P.A.Marchetti, "Bosonization and Duality in Condensed Matter Systems" hep-th/9511100

[10] E.C.Marino, Ann. of Phys. (NY) 224 (1993) 225 
[11] E.C.Marino, "Charge and Magnetic Flux Correlations in Chern-Simons Theory with Fermions" hep-th/9603020

[12] E.C.Marino, Int. J. Mod. Phys. A9 (1994) 4009

[13] E.C.Marino, Nucl. Phys. B408[FS] (1993) 551

[14] L.P.Kadanoff and H.Ceva, Phys. Rev. B3 (1971) 3918

[15] R.L.P.G. do Amaral and E.C.Marino, J. Phys. A25 (1992) 5183

\section{Figure Captions}

Fig. 1 - Surface used in the definition of the $\mu$-operator.

Fig. 2 - Parameters characterizing $\left|\xi-x_{i}\right|$. Notice that $\alpha=\arg \left(x_{i}-y_{j}\right)$ and $\theta=\arg \left(\xi-y_{j}\right)$. 